\title{
X-Ray Spectroscopy of Gamma-Ray Bursts
}

\author{
L. Piro \\ Istituto Astrofisica Spaziale Fisica Cosmica, CNR, Roma, Italy
}

\begin{abstract}
Observational evidence of iron absorption and emission lines in X-ray spectra of Gamma-Ray Bursts is quite compelling. I will briefly review the results, summarize different models and describe the connection with massive progenitors in star-forming regions implied by these results. This link is also supported by measurements of the X-ray absorbing gas in several GRB's, with column density consistent with that of Giant Molecular Clouds harbouring star-formation in our Galaxy, as well as by evidences gathered in other wavelengths. However, the volume density inferred by the fireball-jet model is much lower than typical of a GMC, and I will confront this with the alternative explanation of fireball expansion in a high dense medium, outlining the problems that both models have at present. Finally I will briefly summarize some results on dark GRB's, and describe the prospects of high resolution X-ray spectroscopy in getting closer to the central environment of GRB, and far in the Early Universe by using GRB as beacons to probe star and galaxy formation.
\end{abstract}

\section{INTRODUCTION}

X-ray observations of GRB are playing a key role in several areas of the GRB research. Most of the fast and precise localizations of GRB have been obtained in X-rays by BeppoSAX, HETE-2 and Rossi XTE by combining a gamma-ray burst monitor trigger with the location accuracy provided by X-ray detectors.

$\mathrm{X}$-ray spectroscopy of the prompt and afterglow emission is providing important clues as to the origin of GRB progenitors, the emission processes and the environment of different classes of GRB. The presence of emission and absorption Fe X-ray lines in some GRB is indicating massive progenitors in star forming regions. The gas in the local environment of the GRB or in the host galaxy affects also the X-ray spectral shape. In this regard, Xray spectral measurements can provide information on the column density of the gas, its chemical composition and ionization stage.

The characterization of the environment is of particular importance to understand the origin of different classes of GRB's: optically bright and dark GRB's, Xray rich GRB, and short GRB's. Some of the differences amongst two or more classes could be in fact the result of a different environment, rather that reflecting intrinsic differences in the central source. While this could not be the case of short GRB (thought to be produced by binary mergers rather than by massive progenitors [e.g. 1] $)^{1}$, this sort of unification scenario of GRB could in principle account for other classes properties. For example, dark events could be associated to GRB whose optical light is effectively estinguished by dust either in the local environment or in the host galaxy [e.g. 2]. X-ray rich events could be associated with "dirty" fireballs [3], i.e. fireballs that, due to high baryon loading, expand with a much lower Lorentz factor, boosting the photons in the X-ray band rather than in the $\gamma$-ray range.

Another important piece of information that could play an important role in making up different observed properties is the distance. For example the properties of $\mathrm{X}$-ray flashes and some dark GRB's can be explained if they lie at $z>5-10$.

In this paper I will review the present picture and discuss future perspectives in assembling some of the missing elements, outlining, in particular, the role of Xray spectroscopy.

\footnotetext{
${ }^{1}$ In this case the environment would make a difference in the afterglow properties because, lacking an external medium to make an external shock, the afterglow would be much fainter than in the case of long bursts
} 

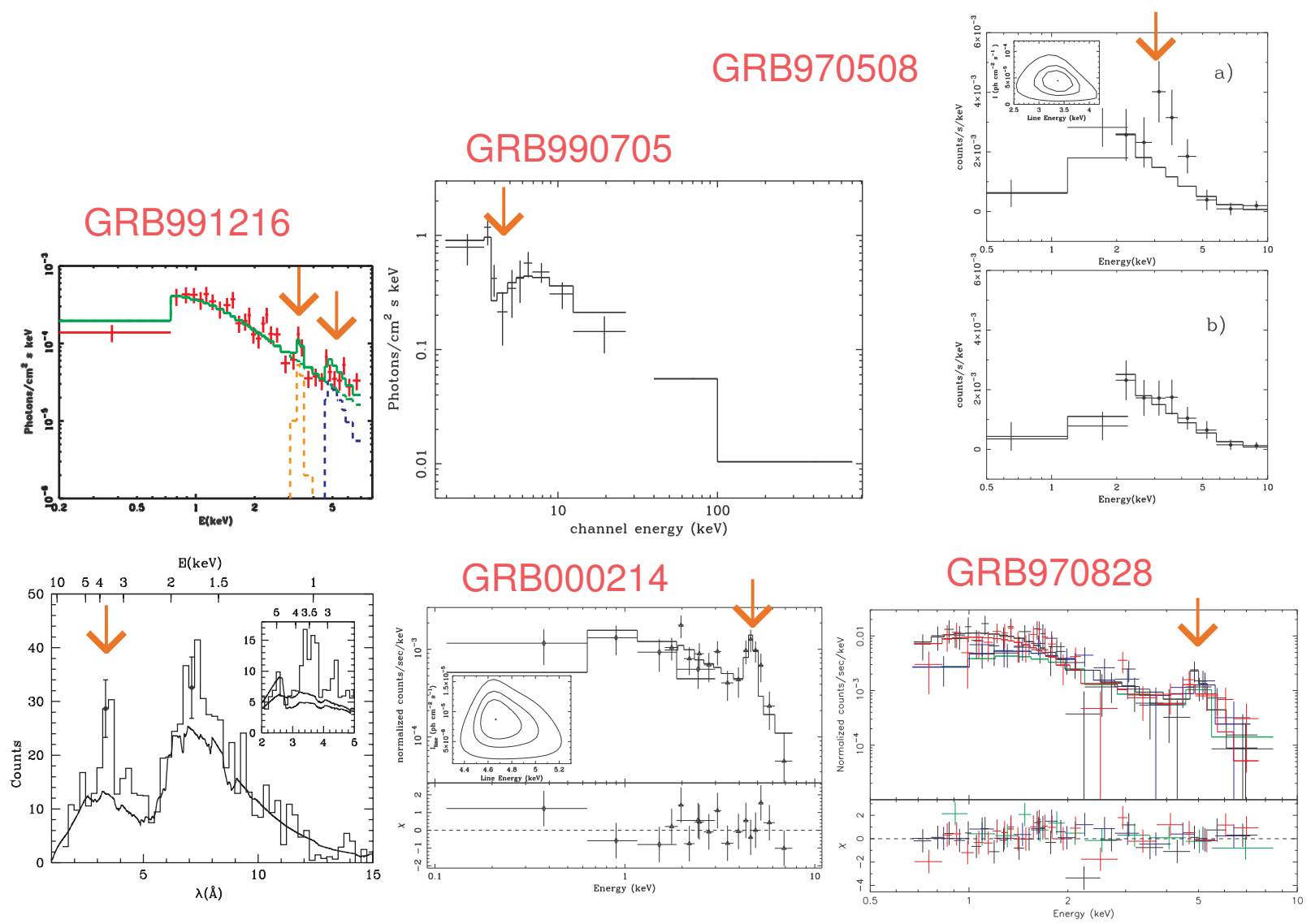

FIGURE 1. Iron features in GRB: GRB970508 [4]; GRB970828: [5]; GRB990214 [6]; GRB991216 [7]);GRB990705 [8])

\section{X-RAY ABSORPTION AND EMISSION FEATURES}

Iron features are ubiquitous in several classes of X-ray sources and have been used to probe the emission mechanisms and the close environment of these sources[9]. The observational evidence of iron features in the X-ray spectra of GRB is quite compelling. So far, there have been 5 detections of iron features in GRB (Fig.1), four in the afterglow phase (GRB970508 [4]; GRB970828: [5]; GRB990214 [6]; GRB991216 [7]), and one during the prompt phase (GRB990705 [8]), each at about 3 sigma level (with the exception of GRB991216, with a significance above 4 sigma). In three cases (GRB970508, GRB970828, GRB991216), the X-ray redshifts derived from the iron lines were consistent with those from optical spectroscopy. For the dark GRB000214, no optical redshift is available as yet. Finally, the case of GRB990705 is particularly important. No optical spectroscopy was available when the original paper was published[7]. The energy of the iron feature implied an $\mathrm{X}$-ray redshift $z=0.86 \pm 0.14$. This prediction is now confirmed by optical spectroscopy, that set the redshift of the host galaxy at $z=0.8435$ [10]. These findings demonstrate that the measurement of the redshift by Xray spectroscopy is more than a mere possibility, and that it can provide reliable results, a fact that is particularly important when optical spectroscopy is difficult or not possible at all.

Let us now summarize the observational picture and the ensuing theoretical implications. For the features in the three afterglows with an independent optical redshift, we can derive the line energy at the burst site and therefore the ionization stage of the medium. FeI to Fe XVII ions are characterized by a $K_{\alpha}$ line energy at $\approx 6.4 \mathrm{keV}$, that rises to $6.7 \mathrm{keV}$ for He-like ions and to $6.9 \mathrm{keV}$ for $\mathrm{H}$-like ions. The iron edge is located at $7.1 \mathrm{keV}$ for neutral iron, rising slowly to $8.8 \mathrm{keV}$ for He-like ions, and then to $9.3 \mathrm{keV}$ for $\mathrm{H}$-like ions.

In GRB991216 ( $\mathrm{z}=1.02)$ the rest-frame line energy is $6.95 \pm 0.15$. There is also evidence of an additional emission feature at $9 \pm 1 \mathrm{keV}$, that is associated with a narrow $\mathrm{H}$-like iron recombination edge in emission (also known as Radiative Recombination Continuum, RRC). In the 
case of GRB970828 ( $\mathrm{z}=0.96)$, the emission feature at $9.3 \pm 0.5$ has been also attributed to the H-like recombination edge in emission. In the case of GRB970508, the line energy is $6.25 \pm 0.55$, i.e. consistent with an iron stage from neutral to H-like.

There is also marginal evidence of line variability on time scales of $\lesssim d a y$ in three afterglows (GRB970508, GRB970828 and GRB000214), while for GRB991216 the observation was too short $(10 \mathrm{ksec})$. In the latter case a line broadening of $\approx 10 \%$ is measured.

The first conclusion that can be drawn is that the lineemitting gas, in the afterglow phase, is highly ionized. The most obvious source of ionization is the GRB itself, that produces a copious flux of hard X-ray photons (photoionization scenario). In this setting, the medium has to be located outside the fireball region (i.e. at $R \gtrsim 10^{15} \mathrm{~cm}$ : distant reprocessor scenario[e.g. 11, 7, 12]). The line variability is naturally expected from the light travel time between the GRB and the reprocessor.

In the early phase (i.e. on a time scale of $\approx 10 \mathrm{~s}$ ), when the ionization front is still expanding, a substantial fraction of the medium in the line of sight is still to be ionized, thus producing X-ray absorption features, that will disappear when the medium is becomes completely ionized [e.g. 13, 14]. A transient iron absorption edge is then expected if the medium lies in the line of sight, exactly what has been observed in GRB990705. On longer time scales, $\left(\approx t_{\text {rec }}\right.$, the recombination time scale), electrons recombine with ions, producing the line and the RRC [e.g. 15, 7], observed in the afterglow phase. This scenario requires the presence of a dense medium with high iron overabundance ejected before GRB, with a velocity (implied by the line width) of $\approx 0.1 c$ and a mass of iron $\gtrsim 0.01 \mathrm{M}_{\odot}$ [e.g. 7]. The most straightforward scenario that emerges is that of a massive progenitor that ejects, before the GRB, a substantial fraction of its mass in a SN-like explosion, as in the case of the SupraNova model [16].

In the (nearby reprocessor scenario $[17,18]$ ) ionizing photons would be produced by post-burst activity of the "remnant" of the central source after the GRB event. In this case the reprocessor, associated with the outer stellar envelopes of a massive progenitor, can be much nearer to the central source. Line variations are caused by the decaying radiation continuum of the remnant. While a massive progenitor is still assumed in this scenario, an highly iron-enriched medium (and therefore the associated SN event) is no more required, because of the higher densities and the greater reprocessing efficiency in producing a line with the requested luminosity $\left(L_{F e} \approx 10^{44-45} \mathrm{ergs}^{-1}\right)$ [see 19 , for a comparative discussion on the reprocessor scenario].

Finally, in the shock heated scenario, the gas is collisionally ionized by shock resulting from the interaction of GRB ejecta (either the fireball itself or the non- relativistic ejecta of $\mathrm{SN}$-like explosion associated with the GRB) with the material pre-ejected before the GRB by massive progenitor systems [e.g. 20, 21, 22]. The presence of an iron recombination edge in emission is not straightforwardly explained in this scenario, because in the aforementioned models the plasma is expected to be near to thermal equilibrium. However Yonetoku et al. [23] argue that, under certain conditions, a plasma in a non-equilibrium state (NEI) can also produce a narrow RRC.

\section{STAR FORMING REGIONS, X-RAY ABSORPTION AND HIGH-DENSITY ENVIRONMENT}

As discussed above, the presence of X-ray lines links long GRB to massive progenitors, and therefore to starforming sites. There is further independent evidence supporting this connection. In the case of NS-NS coalescence, a substantial fraction of events should take place far from the center of the host galaxy, while the opposite applies to massive progenitors. Bloom et al. [24] have measured the distribution of the offsets of optical afterglows with respect to their host galaxy, finding that it is fully consistent with that of star-forming regions, while the delayed merging scenario can be ruled out at the $2 \times 10^{-3}$ level.

What are the implications of this scenario in terms of observable quantities? In a typical Giant Molecular Cloud harboring star formation in our Galaxy, densities are $n \approx 10^{2}-10^{5} \mathrm{~cm}^{-3}$, the size is of order of $10 \mathrm{pc}$ and the column density is $N_{H} \approx 5-10 \times 10^{21} \mathrm{~cm}^{-2}[25,26]$.

Such values of column density can be measured by current X-ray satellites in relatively bright $\mathrm{X}$-ray afterglows with $z \lesssim 3$ (note in fact that $N_{\text {Hobs }} \approx(1+z)^{-8 / 3} N_{\text {Hrest }}$ and the typical uncertainty on $N_{H}$ obs is $\approx 10^{20} \mathrm{~cm}^{-2}$ ). This is in fact the case. In the BeppoSAX sample at least three afterglows (GRB980703 [27, 28, 29]; GRB010222 [30, 28, 29] and GRB990123[28, 29]), show significant absorption, and two other some marginal evidence. $N_{\text {Hrest }}$ in the range $(2-20) 10^{21} \mathrm{~cm}^{-2}$. In addition, Chandra (and BeppoSAX) observations of two other afterglows give similar results $\left(\right.$ GRB000210: $N_{\text {Hrest }}=(5 \pm 1) 10^{21} \mathrm{~cm}^{-2}$ [31]; GRB000926: $\left.N_{\text {Hrest }}=\left(4_{-2.5}^{+3.5}\right) 10^{21} \mathrm{~cm}^{-2}[32]\right)$. These measurements of absorption provide further support to the scenario in which GRB are embedded in a star-forming GMC, in which the typical volume density is $n \approx 10^{2-5} \mathrm{~cm}^{-3}$.

However, application of the standard fireball-jet model to multi-wavelength data of afterglows leads to density estimates that are typically much lower that that expected in a GMC [e.g. 33]. There are two points that need 
to be stressed in this regard. First, the achromatic (i.e. energy-independent) break observed in the light curve of some afterglows is attributed to a collimated fireball. The break appears when the relativistic beaming angle $1 / \Gamma$ becomes $\approx \theta$ (e.g. Rhoads 1997, Sari et al.1999). The typical opening angle of the jet derived in these models is of $\approx 2-4^{\circ}$ [e.g. 33, 34]. We have performed a systematic analysis of the X-ray spectra and light curves on a sample of BeppoSAX afterglows observed from few hours to about 2 days after the GRB [35] that shows that the fireball expansion in the first two days is consistent with a spherical expansion. This result implies that the average opening angle of the jet should be $\theta \gtrsim 10-20^{\circ}$. As for a probable origin of the discrepancy we note that in [e.g. 33] the X-ray data are considered at just one energy, overlooking the fact that the X-ray window span almost two decades in energy, and therefore not including the $X$-ray spectral information.

For example, in the case of GRB010222, where a break in the light curves appears around 0.5 days [30, 36], the predictions of the standard jet model are not consistent with the X-ray temporal and spectral slopes [30]. On the contrary, the X-ray data are well described by a fireball undergoing a transition to a non-relativistic expansion (NRE, [37]). In fact, NRE can also produce an achromatic break in the afterglow light curves at $t_{N R E} \approx 3 \frac{1+z}{2}\left(\frac{E_{53}}{n_{6}}\right)^{1 / 3}$ days. This alternative explanation has been proposed in a few other cases (GB990123 [38], GRB000926 [32] - but see also[39] , GRB010222 $[30,36]$. A strong implication of this scenario is that, in those GRB's, the environment is composed of a dense medium $\left(n \approx 10^{4}-10^{6} \mathrm{~cm}^{-3}\right.$ ), i.e. typical of molecular clouds in star forming regions. Interestingly, in the case of GRB010222, a strong, constant sub-mm emission, has been attributed to enhanced star-forming activity $(\approx 600$ $\left.\mathrm{M}_{\odot} \mathrm{yr}^{-1}\right)$ in the galaxy hosting this burst[40]. Such high densities require a low magnetic field, $\lesssim 10^{-6}$ times the equipartition value, to keep the synchrotron selfabsorption frequency in the observed region. Comparable values of the magnetic field have been also derived in other cases, like GRB971214 [41] and GRB990123 [42]. However, a first attempt to fit the broad-band data of GRB000926 with the NRE show that the radio data are not well described by the model [39].

In conclusion, at the present stage of analysis, both the NRE of a moderately-collimated fireball in a dense medium and the highly collimated jet scenario (in a low density medium) show some inconsistency with the data, that appears when the complete information from radio to X-rays is considered. More theoretical efforts are thus needed, in particular to reconcile the fireball model with an external medium typical of a star-forming region.

\section{RECENT FINDINGS AND REMAINING MYSTERIES}

\section{GHOST and X-ray flashes}

It is observationally well-established that about half of accurately localized gamma-ray bursts (GRBs) do not produce a detectable optical afterglow [43, 44], while most of them $(\approx 90 \%)$ have an X-ray afterglow [45]. Statistical studies have shown that the optical searches of these events, known variously as "dark GRBs", "failed optical afterglows" (FOA), or "gamma-ray bursts hiding an optical source-transient" (GHOST), have been carried out to magnitude limits fainter on average than the known sample of optical afterglows $[46,2]$. Thus dark bursts appear to constitute a distinct class of events and are not the result of an inadequate search, but it is unclear whether this observational property derives from a single origin or it is a combination of different causes.

If the progenitors of long-duration GRBs are massive stars [47], as current evidence suggests (e.g., [48, 7]), extinction of optical flux by dusty star-forming regions is likely to occur for a substantial fraction of events (the obscuration scenario[e.g. 2])). Another possibility is that dark GRBs are located at redshift $z \gtrsim 5$, with the optical flux being absorbed by the intervening Ly $\alpha$ forest clouds (the intergalactic scenario [e.g. 49]).

Dark bursts which can be localized to arcsecond accuracy, through a detection of either their X-ray or radio afterglow, are of particular interest. The first example is GRB 970828 for which prompt, deep searches down to $\mathrm{R} \sim 24.5$ failed to detect an optical afterglow [50, 51] despite it was localized within a region of only 10 arcsec radius by the ROSAT satellite [52]. Djorgovski et al. [53] recently showed how the detection of a short-lived radio transient for GRB 970828 allowed them to identify the probable host galaxy and to infer its properties (redshift, luminosity and morphology). In addition, they used estimates of the column density of absorbing gas from X-ray data, and lower limits on the rest frame extinction $\left(\mathrm{A}_{V}>3.8\right)$ to quantify the amount of obscuration towards the GRB. The other best studied example is GRB000210 [31]. This burst had the highest $\gamma$-ray peak flux of any event localized by BeppoSAX as yet but it did not have a detected optical afterglow, despite prompt and deep searches down to $R_{\text {lim }} \approx 23.5$. Chan$d r a$ observations allowed to localize the X-ray afterglow of GRB000210 to within $\approx 1$ arcsec and a radio transient was detected with the VLA. The precise X-ray and radio positions allowed to identify the likely host galaxy of this burst, and to measure its redshift, $z=0.846$. The probability that this galaxy is a field object is $\approx 10^{-2}$, but the chance that this happens in both the cases of GRB970828 \& GRB000210 is negligible. 

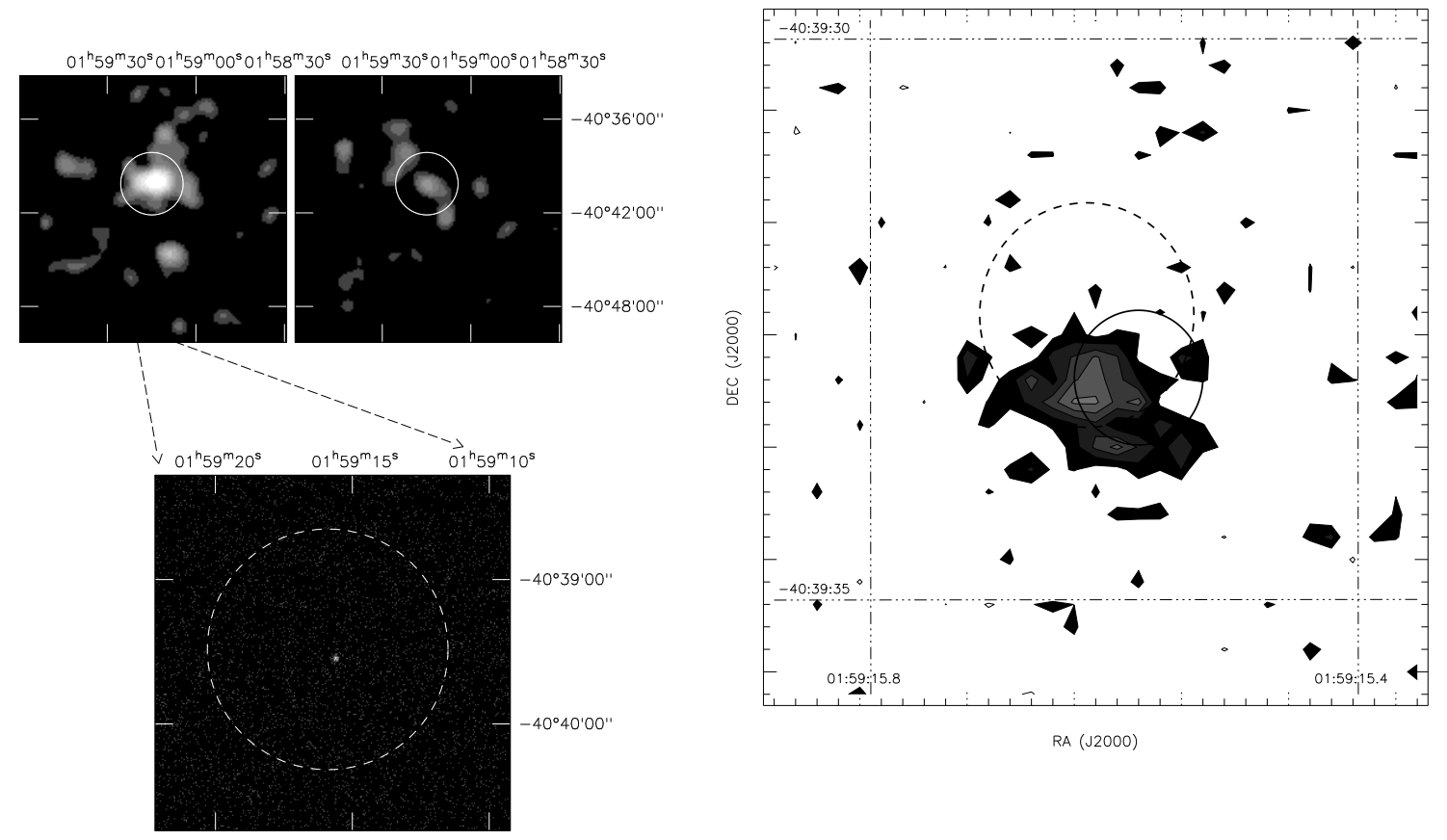

FIGURE 2. The dark GRB000210. The two images in the left upper panel show the decaying X-ray afterglow BeppoSAX MECS(1.6-10 keV) $8 \mathrm{hrs}$ and $30 \mathrm{hrs}$ after the GRB respectively. The circle is the WFC error box. The left lower panel is the Chandra ACIS-S image of the afterglow $21 \mathrm{hrs}$ after the GRB. The dashed line is the BeppoSAX MECS error box. The right panel shows the optical image of the host galaxy taken with the VLT. The circles show the $90 \%$ error circles of the Chandra (continuous line) and radio (dashed line) afterglows. The redshift of the galaxy is $\mathrm{z}=0.846$

The X-ray spectrum of the afterglow of GRB000210 shows significant absorption in excess of the Galactic one corresponding, at the redshift of the galaxy, to $N_{H}=$ $(5 \pm 1) \times 10^{21} \mathrm{~cm}^{-2}$. The amount of dust needed to absorb the optical flux of this object is consistent with the above HI column density, given a dust-to-gas ratio similar to that of our Galaxy. If the absorption takes place in a GMC, a substantial fraction of the X-ray gas should be heavily ionized by the hard X-ray photons emitted by the GRB and its afterglow. This effect should change the absorption profile, in particular at lower energies, where the lighter elements are heavily ionized, and therefore become more transparent to the radiation. Nonetheless, the X-ray absorption observed in GRB000210 is consistent with a cold medium, an evidence that can be reconciled with the GMC scenario is the medium is condensed in high dense clouds $\left(n \gtrsim 10^{9} \mathrm{~cm}^{-3}\right.$ [31].

Given the extreme luminosity of GRBs and their probable association with massive stars, it is expected that some fraction of events will be located beyond $z>5$ [54]. These would be probably classified as dark bursts because the UV light, which is strongly attenuated by absorption in the $\mathrm{Ly}_{\alpha}$ forest, is redshifted into the optical band. We note that the four redshifts determined or suggested so far for dark GRBs $(z=0.96$, GRB970828, [53]; $z=1.3$, GRB990506, [55, 24]; $z \approx$
0.47, GRB000214, [6], GRB000210 [31]) are in the range of those measured for most bright optical afterglows, but whether this applies to the majority of these events is still to be assessed.

Particularly interesting in this respect is the case of the so-called X-ray flashes or X-ray rich GRBs discovered by BeppoSAX[56, 57]. No optical counterpart has been identified as yet in any of these bursts. The intergalactic scenario would naturally explain both the absence of optical afterglows and the high-energy spectrum, because the peak of the gamma-ray spectrum would be redshifted into the X-ray band. Alternatively, the paucity of gammaray emission can be explained by a "dirty" fireball [3]. In such a case the fireball will achieve a Lorentz factor much lower than previously considered, i.e. not high enough to boost the photons in the $\gamma$-ray range.

\section{Short GRB's}

The distribution of GRB duration appears to be bimodal, with about $30 \%$ of events lasting less than $1 \mathrm{sec}$ [58]. It is still unclear whether these events are intrinsically diverse from long bursts, i.e. if they are produced by different progenitors. So far, very little is known about these events, due to the lack of a counterpart. While the 
GRBM on board of BeppoSAX is detecting those events, no one has so far been observed in the X-ray range (and therefore precisely localized) by the WFC [59]. The probability that this is a chance fluctuation is getting interestingly low, suggesting a depleted X-ray emission compared to the class of long events [60]. If that is the case, the localization of afterglows of these events should rely only on experiments with good position accuracy in the $\gamma$-ray range, like the currently operating IPN or, in the future, SWIFT. If, instead, these events have an X-ray emission similar to long ones, we may hope to localize a few of them with BeppoSAX and HETE2.

\section{THE FUTURE OF X-RAY SPECTROSCOPY}

$\mathrm{X}$-ray spectroscopy of GRB is coming of age. Emission and absorption features as well as the properties of $\mathrm{X}$ ray absorption are providing key information on the close environment of long GRB's, suggesting massive progenitors and a connection between these events and starforming regions. The possibility of measuring the redshift directly from X-rays is of particular value for those classes of objects, like the majority of dark GRB, X-ray flashes and short GRB's, that still lack optical counterparts. The radiation intensity of GRB's is so high that they can be detectable out to much larger distances than those of the most luminous quasars or galaxies observed so far, and it is likely that high-z GRB are actually the constituents of one of those mysterious classes of events.

We should stress that, with present X-ray facilities (BeppoSAX, XTE, HETE2, Chandra, Newton), the progress in this field will follow "quantum" jumps. In fact, to get good-quality X-ray spectra, one should catch bright afterglows, but these should be not too many, because of the combination of ingredients needed (number of precise and fast locations, reaction time, number of TOO observations allocated to GRB programs). On the positive side, one can then hope that the next important discovery just lies behind the corner.

In the near future, we expect a further advancement in this area, when high quality X-ray CCD spectra of afterglows $(E / \Delta E \approx 50)$ will be routinely available with SWIFT. Looking ahead in the future, high resolution $\mathrm{X}$ ray spectroscopy $(E / \Delta E \gtrsim 1000$, like that provided by $\mathrm{X}$-ray microcalorimeters) should open a new area of exploration $([61,62])$, that would bring us closer to the central engine of GRB, its environment and far in the Early Universe by using GRB as beacons to probe star and galaxy formation. We can foresee the possibility of resolving in detail line profiles, deriving information on the kinematics of the ejecta, or looking for narrow and faint emission lines, imprinted on the spectra by the low- velocity medium embedding the GRB. The measurement of the absorption edges produced by the ISM of the host galaxy will provide information on the chemical composition of galaxies in the Early Universe, thus opening the possibility to trace the metallicity history of the gas in the Universe and to probe the formation of the first stars $[62,63,54]$.

\section{ACKNOWLEDGMENTS}

BeppoSAX is a program of the Italian space agency (ASI) with participation of the Dutch space agency (NIVR)

\section{REFERENCES}

1. Woosley, S. E., in GRBs in the Afterglow Era, edited by E. Costa, F. Frontera, and J. Hjorth, ESO-Springer, 2001, pp. 258-262.

2. Reichart, D. E., and Yost, S. A., (2001), apJ, in press; astro-ph/0107545.

3. Dermer, C. D., Chiang, J., and Boettcher, M., ApJ, 513, 656-668 (1999).

4. Piro, L., et al., A\&A, 331, L41-L44 (1998).

5. Yoshida, A., Yonetoku, N. M., Murakami, T., Otani, C., Kawai, N., Ueda, Y., Shibata, R., and Uno, S., ApJ, 557, L27-L30 (2001).

6. Antonelli, L. A., Piro, L., Vietri, et al.ApJ, 545, L39-L42 (2000).

7. Piro, L., Garmire, G., Garcia, et al.Science, 290, 955-958 (2000).

8. Amati, L., Frontera, F., Vietri, et al.Science, 290, 953-955 (2000).

9. Piro, L., in $U V$ and $X$-ray spectroscopy of Laboratory and Astrophysical Plasmas, edited by S. K. E. Silver, Cambridge Uni. Press, 1993, p. 448.

10. Andersen, M., et al. (2002), in preparation.

11. Lazzati, D., Campana, S., and Ghisellini, G., MNRAS, 304, L31 (1999).

12. Weth, C., et al., ApJ, 534, 581 (2000).

13. Perna, R., and Loeb, A., ApJ, 501, 467- (1998).

14. Boettcher, M., Dermer, C. D., Crider, A. W., and Liang, E. . P., $A \& A$, 343, 111-119 (1999).

15. Paerels, F., Kuulkers, E., Heise, J., and Liedahl, D. A., ApJ, 535, L25-L28 (2000).

16. Vietri, M., and Stella, L., ApJ, 527, L43-L46 (1999).

17. Rees, M. J., and Mészáros, P., ApJ, 545, L73-L75 (2000).

18. Mészáros, P., and Rees, M. J., ApJ, 556, L37-L40 (2001).

19. Kallman, T. R., Mészáros, P., and Rees, M. J., Iron k lines from grb (2002), astro-ph/00110654.

20. Vietri, M., Perola, G. C., Piro, L., and Stella, L., MNRAS, 308, L29 (1999).

21. Boettcher, M., ApJ, 539, 102-110 (2000).

22. Boettcher, M., and Fryer, C. L., ApJ, 547, 338-344 (2001).

23. Yonetoku, N. M., Murakami, T., Masai, K., Yoshida, A., Kawai, N., and Namiki, M., ApJ, 557, L23-L26 (2001). 
24. Bloom, J. S., Kulkarni, S. R., and Djorgovski, S. G.(2002), aJ, in press; astro-ph/0010176.

25. Balsara, D., Ward-Thompson, D., and Crutcher, R. M., MNRAS, 327, 715-720 (2001).

26. Ward-Thompson, D., Scott, P. F., Hills, R. E., and Andre, P., MNRAS, 268, 276-290 (1994).

27. Vreeswijk, P. M., et al.ApJ, 523, 171-176 (1999).

28. Pasquale, M. D., et al. (2002), in preparation.

29. Stratta, G., et al. (2002), in preparation.

30. in' t Zand, J. . J. M., Kuiper, L., Amatiet al.ApJ, 559, 710-715 (2001)

31. Piro, L., Frail, D., Gorosabel, J., et al.(2002), apJ, submitted; astro/ph-0201282.

32. Piro, L., Garmire, G., Garcia, et al.ApJ, 558, 442-447 (2001).

33. Panaitescu, A., and Kumar, P., ApJ, 554, 667-677 (2001).

34. Frail, D. A., et al., ApJ, 562, L55- (2001).

35. Stratta, G., Piro, L., Soffitta, P., et al., in GRBs in the Afterglow Era, edited by E. Costa, F. Frontera, and J. Hjorth, ESO-Springer, 2001, pp. 118-120.

36. Masetti, N., et al., $A \& A, \mathbf{3 7 4}, 382$ (2001).

37. Livio, M., and Waxman, E., ApJ, 538, 187-191 (2000).

38. Dai, Z. G., and Lu, T., ApJ, 519, L155-L158 (1999).

39. Harrison, F. A.,et al.559, 523 (2001).

40. Frail, D. A., et al.(2001), submitted to ApJ; astro$\mathrm{ph} / 0108436$.

41. Wijers, R. A. M. J., and Galama, T. J., ApJ, 523, 177-186 (1999).

42. Galama, T. J., et al., Nature, 398, 394-399 (1999).

43. Frail, D. A., Kulkarni, S. R., Wieringa, M. H., Taylor, G. B., Moriarty-Schieven, G. H., Shepherd, D. S., Wark, R. M., Subrahmanyan, R., McConnell, D., and Cunningham, S. J., , in AIP Conf. Proc. 526: Gamma-ray Bursts, 5th Huntsville Symposium, 2000, pp. 298-302.

44. Fynbo, J. U., et al.A\&A, 369, 373-379 (2001).

45. Piro, L., in GRBs in the Afterglow Era, edited by E. Costa, F. Frontera, and J. Hjorth, ESO-Springer, 2001, pp. 97-105.

46. Lazzati, D., Covino, S., and Ghisellini, G., (2002), mNRAS, in press, astro-ph/0011443.

47. Paczyński, B., ApJ, 494, L45-L48 (1998).

48. Bloom, J. S., et al., Nature, 401, 453-456 (1999).

49. Fruchter, A. S., ApJ, 512, L1-L4 (1999).

50. Odewahn, S. C., et al.IAU Circ, 6735 (1997).

51. Groot, P. J.et al.ApJ, 493, L27-+ (1998).

52. Greiner, J., Schwarz, R., Englhauser, J., Groot, P. J., and Galama, T. J., IAU Circ, 6757 (1997).

53. Djorgovski, S. G., , Frail, D. A., Kulkarni, S. R., Bloom, J. S., Odewahn, S. C., and Dierks, A., ApJ, 562, 654 (2001).

54. Lamb, D. Q., and Reichart, D. E., ApJ, 536, 1-18 (2000).

55. Taylor, G. B., Bloom, J. S., Frail, D. A., Kulkarni, S. R., Djorgovski, S. G., and Jacoby, B. A., ApJ, 537, L17-L21 (2000).

56. Heise, J., in 't Zand, J., Kippen, M., and Woods, P., in GRBs in the Afterglow Era, edited by E. Costa, F. Frontera, and J. Hjorth, ESO-Springer, 2001, pp. $16-21$.

57. Heise, J. (2002), this conference.

58. Kouveliotou, C., Meegan, C. A., Fishman, G. J., Bhat, N. P., Briggs, M. S., Koshut, T. M., Paciesas, W. S., and Pendleton, G. N., ApJ, 413, 101-104 (1993).

59. Gandolfi, G., et al., in AIP Conf. Proc. 526: Gamma-ray
Bursts, 5th Huntsville Symposium, edited by M. Kippen, R. Mallozzi, and G. J. Fishman, 2000, pp. 23-.

60. Gandolfi, G., et al. (2002), this conference.

61. Piro, L., et al.,in GRBs in the Afterglow Era, edited by E. Costa, F. Frontera, and J. Hjorth, ESO-Springer, 2001, p. 415.

62. Piro, L. (1999), probing the Early Universe with GRB and XEUS at www.ias.rm.cnr.it/sax/xeus.html.

63. Fiore, F., et al., ApJ, 544, L7- (2000). 